\title{
Author Index Volume 26
}

Abdelkafi, O., Idoumghar, L. and Lepagnot, J., A survey on the metaheuristics applied to QAP for the graphics processing units

26 (2016) 1650013

Akl, S. G., Editorial note

26 (2016) 1601001

Akl, S. G., Editorial note

26 (2016) 1601002

Akl, S. G., Editorial note

26 (2016) 1601003

Akl, S. G., Editorial note

26 (2016) 1601004

Applebaum, B., Kowalski, D. R., Patt-Shamir, B. and Rosén, A., Clique here: On the distributed complexity in fully-connected networks

26 (2016) 1650004

Aupy, G. and Benoit, A., Approximation algorithms for energy, reliability, and makespan optimization problems

26 (2016) 1650001

Bandyopadhyay, S., Sarkar, D., Mandal, C., Banerjee, K. and Duddu, K. R., A path construction algorithm for translation validation using PRES+ models

Banerjee, K., see Bandyopadhyay, S.

Benoit, A., see Aupy, G.

Bruda, S. D. and Wilkin, M. S. R., Limitations of coverability trees for context-free parallel communicating grammar systems and why these grammar systems are not linear space

Chen, C., see Fang, J.

Chen, X., see Fang, J.

Cheng, E., Qiu, K. and Shen, Z., On the conditional diagnosability of hyper-butterfly graphs and related networks

Cohen, J., Lefèvre, J., Maâmra, K., Pilard, L. and Sohier, D., A self-stabilizing algorithm for maximal matching in anonymous networks

Crain, T., Gramoli, V. and Raynal, M., A fast contention-friendly binary search tree

26 (2016) 1650015

26 (2016) 1650010

Fang, J., Zhang, P., Li, Z., Tang, T., Chen, X., Chen, C. and Yang, C., Evaluating multiple streams on heterogeneous platforms

26 (2016) 1640002

Flatz, M. and Vajteršic, M., Parallel nonnegative matrix factorization via Newton iteration

26 (2016) 1650014 
Gao, J., Zhou, Y. and Wu, K., A novel multi-GPU parallel optimization model for the sparse matrix-vector multiplication

Gijsbers, B., see Zaichenkov, P.

Gramoli, V., see Crain, T.

Grelck, C., see Zaichenkov, P.

Higham, L. and Kawash, J., Pitfalls in memory consistency modelling

Iacono, M. J., see Michalakes, J.

Idoumghar, L., see Abdelkafi, O.

Imbs, D. and Raynal, M., Trading off $t$-resilience for efficiency in asynchronous Byzantine reliable broadcast

Jaja, J., see $\mathrm{Wu}, \mathrm{J}$.

Jessup, E. R., see Michalakes, J.

Kawash, J., see Higham, L.

Kent, K. B., see Wang, Y.

Kowalski, D. R., see Applebaum, B.

Kumar Chande, M., Lee, C.-C. and Li, C.-T., A new self-certified convertible authenticated encryption scheme based on discrete logarithm problem

Kurmas, Z., see Trefftz, C.

Lee, C.-C., see Kumar Chande, M.

Lefèvre, J., see Cohen, J.

Lepagnot, J., see Abdelkafi, O.

Li, C.-T., see Kumar Chande, M.

Li, Z., see Fang, J.

Maâmra, K., see Cohen, J.

Mandal, C., see Bandyopadhyay, S.

Maurer, A. and Tixeuil, S., Tolerating random Byzantine failures in an unbounded network

McGuire, H., see Trefftz, C.

Michalakes, J., Iacono, M. J. and Jessup, E. R., Optimizing weather model radiative transfer physics for Intel's many integrated core (MIC) architecture

Mishra, A. and Mishra, P. K., A randomized scheduling algorithm for multiprocessor environments using local search

Mishra, P. K., see Mishra, A.

Patt-Shamir, B., see Applebaum, B.

Pilard, L., see Cohen, J.

Qiu, K., see Cheng, E.

Raynal, M., see Crain, T.

Raynal, M., see Imbs, D.

Rosén, A., see Applebaum, B.

Sarkar, D., see Bandyopadhyay, S.

Scripps, J., see Trefftz, C.

Shafarenko, A., see Zaichenkov, P.

Shen, Z., see Cheng, E.

26 (2016) 1640001

26 (2016) 1650011

26 (2016) 1650015

26 (2016) 1650011

26 (2016) 1650008

26 (2016) 1650019

26 (2016) 1650013

26 (2016) 1650017

26 (2016) 1650007

26 (2016) 1650019

26 (2016) 1650008

26 (2016) 1650009

26 (2016) 1650004

26 (2016) 1650018

26 (2016) 1650006

26 (2016) 1650018

26 (2016) 1650016

26 (2016) 1650013

26 (2016) 1650018

26 (2016) 1640002

26 (2016) 1650016

26 (2016) 1650010

26 (2016) 1650003

26 (2016) 1650006

26 (2016) 1650019

26 (2016) 1650002

26 (2016) 1650002

26 (2016) 1650004

26 (2016) 1650016

26 (2016) 1650005

26 (2016) 1650015

26 (2016) 1650017

26 (2016) 1650004

26 (2016) 1650010

26 (2016) 1650006

26 (2016) 1650011

26 (2016) 1650005

Shi, W., see Wang, Y.

26 (2016) 1650009 
Sohier, D., see Cohen, J.

Tang, T., see Fang, J.

Tixeuil, S., see Maurer, A.

Trefftz, C., McGuire, H., Kurmas, Z. and Scripps, J., Exhaustive community enumeration in parallel

Tveretina, O., see Zaichenkov, P.

Vajteršic, M., see Flatz, M.

Wang, Y., Shi, W. and Kent, K. B., On optimal scheduling algorithms for well-structured workflows in the cloud with budget and deadline constraints

Wilkin, M. S. R., see Bruda, S. D.

Wu, J. and Jaja, J., Achieving native GPU performance for out-of-card large dense matrix multiplication

$\mathrm{Wu}, \mathrm{K}$., see Gao, J.

Yang, C., see Fang, J.

Zaichenkov, P., Tveretina, O., Shafarenko, A., Gijsbers, B. and Grelck, C., The cost and benefits of coordination programming:

Two case studies in concurrent collections and S-NET

Zhang, P., see Fang, J.

Zhou, Y., see Gao, J.
26 (2016) 1650016

26 (2016) 1640002

26 (2016) 1650003

26 (2016) 1650006

26 (2016) 1650011

26 (2016) 1650014

26 (2016) 1650009

26 (2016) 1650012

26 (2016) 1650007

26 (2016) 1640001

26 (2016) 1640002

26 (2016) 1650011

26 (2016) 1640002

26 (2016) 1640001 